\title{
Influence of CX-reactions on the radiation in the pedestal region at ASDEX Upgrade
}

\author{
R. Dux, M. Cavedon, A. Kallenbach, R. M. McDermott, G. \\ Vogel and the ASDEX Upgrade team \\ Max-Planck-Institut für Plasmaphysik, Garching, Germany \\ E-mail: Ralph.Dux@ipp.mpg.de
}

\begin{abstract}
The radial density profiles of $\mathrm{Ne}^{10+}$ and $\mathrm{Ne}^{8+}$ have been measured with charge exchange recombination spectroscopy in an H-mode discharge in ASDEX Upgrade. When trying to fit the data with an impurity transport code that only takes electronic ionisation and recombination into account, the density of $\mathrm{Ne}^{8+}$ is too low by more than an order of magnitude indicating that an additional recombination mechanism must be at work. We ascribe the missing recombination channel to charge exchange (CX) reactions between neutral deuterium and the impurity ions, which has long been known to be a very efficient recombination reaction. Including the CXreactions yields a good fit of the ionisation balance and delivers the neutral density profile in the pedestal, which is not known from other diagnostics. Here, the CXreactions lead to a change of the ionisation balance on the whole flux surface and the measurement delivers a flux surface averaged neutral density with the exception of the region very close to the X-point. Furthermore, it leads to an increase of the pedestal radiation of neon since the partially ionised stages can emit line radiation. This amounts to an increase of the radiated power of neon inside of the separatrix by a factor of 5. A similar analysis was done for argon in an H-mode discharge dominated by Ar radiation. Only the CX-recombination in the pedestal can explain the radiated power inside the separatrix, which would be too low by a factor of 2.2 without CX. In addition, the radiances of VUV lines from many charge stages are much better fitted when including the CX-recombination. A simple projection of the impact of CX-recombination to the much hotter ITER pedestals shows that for elements up to $\mathrm{Kr}$, a beneficial increase of edge radiated power per core radiated power and of radiated power per central dilution is obtained, while for Xe and especially for $\mathrm{W}$ the effect is weak.
\end{abstract}


Influence of $C X$-reactions on the radiation in the pedestal region at ASDEX Upgrade 2

\section{Introduction}

The optimisation of radiation losses in the pedestal region of $\mathrm{H}$-mode plasmas is an important ingredient to tune the power flux across the separatrix in a fusion reactor. The radiation critically depends on the ionisation balance of the radiating impurity in this region. This ionisation equilibrium, i.e. the distribution of the abundances of each ion stage, is determined by the rates for ionisation and recombination and the radial fluxes and can be calculated with an impurity transport code. It has long been known, that the charge exchange $(\mathrm{CX})$ reaction of neutral hydrogen with an impurity ion

$$
\mathrm{A}^{z+}+\mathrm{H}^{0} \rightarrow \mathrm{A}^{(z-1)+}+\mathrm{H}^{+}
$$

is a very effective recombination process in addition to the electronic channels: radiative and di-electronic recombination, and that even a low fraction of neutral hydrogen $\left(n_{H} / n_{e}\right.$ around $\left.10^{-5}\right)$ leads to strong modifications of the ionisation balance $[1,2,3]$. However, even though CX into excited levels with high main quantum number $n$ are needed to account for the radiation due to transitions starting from such states $[4,5]$ and are actually the basis for the widely used charge exchange spectroscopy (CXRS) using fast neutral beams [6], CX-reactions are usually neglected when calculating the ionisation balance. The main reason for this is that the neutral hydrogen density and the temperature of the neutrals $T_{n}$ are not as readily available as $n_{e}$ and $T_{e}$, but are needed to compute the respective CX rates.

In this study, the ionisation balance of neon has been assessed in an H-mode discharge in ASDEX Upgrade by measuring the density of fully ionised and He-like neon in the confined plasma with CXRS. The measurements and the determination of the densities will be described in sec.2. In sec.3, an analysis with the $1.5 \mathrm{D}$ impurity transport code STRAHL will show that the ionisation balance without CX yields much too low densities of the He-like ion stage. Then, the cross sections for CX are introduced and it is discussed whether CX-recombination in the confined plasma changes the ionisation balance locally in the region with high neutral density or globally on the whole flux surface. In the following, the calculation of flux surface averaged neutral density profiles for the two populations from neutral beam injection and from recycling neutrals is described and it is shown that the inclusion of CX leads to much better agreement with the measured densities. This is shown for a case with low deuterium puffing and a case with a five times higher puff level. In sec.4, the effect of CX on the radiation of neon in the pedestal of this discharge is evaluated and compared with bolometric measurements. CX increases the radiated power inside the separatrix by a factor of 5 . In sec.5, we study the effect of CX-reactions on the radiation of argon in an H-mode plasma, in which the radiation is dominated by argon radiation due to strong Ar puffing. Also here, we see a strong influence of CX on the ionisation balance which leads to an increase of the radiated power by a factor of 2.2 and a much better fit of individual argon lines in the vacuum ultraviolet. A simple projection to the pedestal radiation in ITER is described in sec.6, where considerably stronger radiation in the pedestal region is found for the 
lighter elements up to krypton, while the radiation from xenon and tungsten is almost unchanged. Sec.7 summarises the most important findings of this study.

\section{Measurement of neon densities}

A steady neon puff was applied during the flat top phase of a type-I ELMy Hmode plasma with plasma current $I_{p}=0.8 \mathrm{MA}$, toroidal field $B_{t}=2.5 \mathrm{~T}$, heating power $P=7.7 \mathrm{MW}$, and line averaged density of $7.5 \times 10^{19} \mathrm{~m}^{-3}$. The radial density profiles of $\mathrm{Ne}^{10+}$ and $\mathrm{Ne}^{8+}$ were measured from the centre up to the separatrix using the core and edge systems of the charge exchange recombination spectroscopy (CXRS) at beam 3 of the neutral beam injection (NBI), which was operated in D at an acceleration voltage of $60 \mathrm{keV}$. For $\mathrm{Ne}^{10+}$, the $n=11-10$ transition of $\mathrm{NeX}$ at $524.9 \mathrm{~nm}$ was used while $\mathrm{Ne}^{8+}$ was measured at the $n=10-9$ transition of NeVIII at $606.9 \mathrm{~nm}$, which is accompanied by the $n=13-11$ transition of NeVIII at $606.4 \mathrm{~nm}$.

The spectra were taken with four spectrometers labelled CEZ, CAZ, CMZ, and CNZ in Fig.1, two for each ion. For each ion, one spectrometer was covering the plasma core with about 20 lines-of-sight and one observed the pedestal region with about 10 linesof-sight [7]. All lines-of-sight are oriented in the toroidal direction. During the whole flat top phase, beam 3 was replaced every $250 \mathrm{~ms}$ for a duration of $50 \mathrm{~ms}$ by another beam, which was injected at the opposite side of the torus. This enables a background subtraction at constant heating power to obtain clean spectra containing only the active CX-lines. The optical setup of all spectrometers is absolutely calibrated by illuminating each line-of-sight in the vessel with a calibrated integrating sphere before the start of the experimental campaign. The discharges were performed in the early phase of the campaign where degradation of the in-vessel optics is not an issue.

It is difficult to distinguish with CXRS between the He-like $\mathrm{Ne}^{8+}$ ion and the fully ionised oxygen $\mathrm{O}^{8+}$ since the emission of NeVIII and OVIII are at the same wavelength and the line-width due to the Doppler broadening differs only by $10 \%$. Therefore, the discharges were performed directly after a fresh boronisation of the vessel walls in order to have as low oxygen concentration as possible. Furthermore, the neon puff was started about $0.6 \mathrm{~s}$ after the heating power and D gas puff reached their flat top to allow for a measurement of the density profile of $\mathrm{O}^{8+}$. Since the data collection of one of the spectrometers that measured NeX failed during the first discharge \#37061, the discharge had to be repeated. The oxygen measurement is taken from \#37061 because in \#37062, there is already some recycling neon present in the plasma before the start of the neon puff. All neon measurements are from \#37062.

The evaluation of the density profile from the radiances of the CX-lines has been described in detail in a recent paper [8]. Here, we used a modification of the method, which is called COLRAD in [8]. The first modification is that for oxygen and Helike neon, the effective charge exchange emission rates are no longer taken from the ADAS [9] adf12-files, but are treated like most other ions with data files that come from many evaluations with the ADAS309 code and do not need to be extrapolated (see [8]). 
Influence of $C X$-reactions on the radiation in the pedestal region at ASDEX Upgrade 4
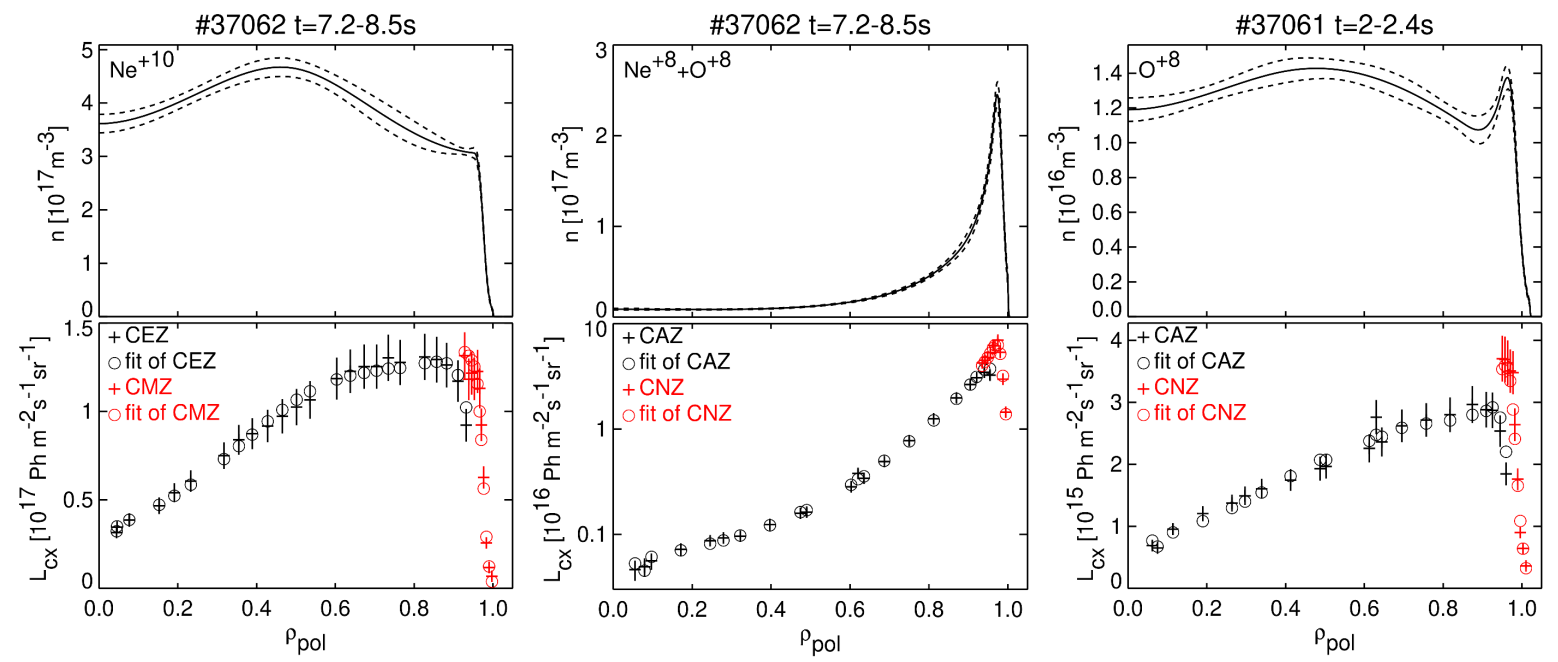

Figure 1. Measured and fitted radiances of the CX-lines on all lines-of-sights (lower row) and corresponding density profiles (upper row) for the measurements of fully ionised neon (left), He-like neon and fully ionised oxygen(middle), and fully ionised oxygen alone (right).

The corresponding ADAS adf-01 files are qcx\#h0_old\#o8.dat for charge exchange from hydrogen in the ground state while for CX from hydrogen in $n=2$ we interpolated the existing qcx\#h0_en2_kvi\#xx.dat-files of the elements from B to Ne to obtain an adf01file for oxygen. The second modification is the treatment of the effect that the plasma parameters including the impurity density vary along the intersection region of neutral beam and line-of-sight, which is especially important in the strong gradient region in the H-mode pedestal. Here, we calculated the beam attenuation for a number of pencils within the beam, such that the local beam densities can be obtained at any point by interpolation. The density profile of the considered impurity ion was parametrised using the exponential of an interpolating spline on the normalised poloidal flux coordinate $\rho_{\text {pol }}$. With this spline function, the line-of-sight integral of the local emissivity can be calculated to obtain the radiance for each chord and allows for a fit of the spline parameters to achieve best agreement with the measured radiances.

The radiances and the corresponding impurity ion density profiles are shown in Fig.1 versus $\rho_{\text {pol }}$. The left column is for $\mathrm{Ne}^{10+}$, the middle for the sum of $\mathrm{Ne}^{8+}$ and $\mathrm{O}^{8+}$, and the right column for $\mathrm{O}^{8+}$. The lower row displays the measured radiances with black symbols for the core spectrometers (CEZ,CAZ) and with red symbols for the edge spectrometers $(\mathrm{CMZ}, \mathrm{CNZ})$. In the plots showing the radiances, $\rho_{\text {pol }}$ is the emissivity weighted average flux label, i.e. $\int \epsilon \rho_{\text {pol }} d l / \int \epsilon d l$, where the integrals are along the line-of-sight in the intersection region with the neutral beam. The line-of-sight effects are clearly visible in the overlap region between the core and edge systems, where the edge lines-of-sight are more tangential to the flux surface and see on average a higher impurity density from the top of the pedestal than the lines-of-sight of the core system which also pass through regions with lower impurity density down from the pedestal 
top and consequently collect less light. The fitted radiances are shown with circles. The uncertainty estimate for the radiances is difficult. When assuming a $10 \%$ uncertainty for the radiances plus an offset value for such cases, where very low and very high signal levels appear on the spectrometer camera as is the case for NeVIII, the normalised $\chi^{2}$ values of the fit are between 0.4 and 0.9 . Thus, there are no systematic deviations in the absolute calibration of the two spectrometers which measure the same emission line. The statistical error leads to a relative uncertainty of the impurity ion densities in the range of $5 \%$.

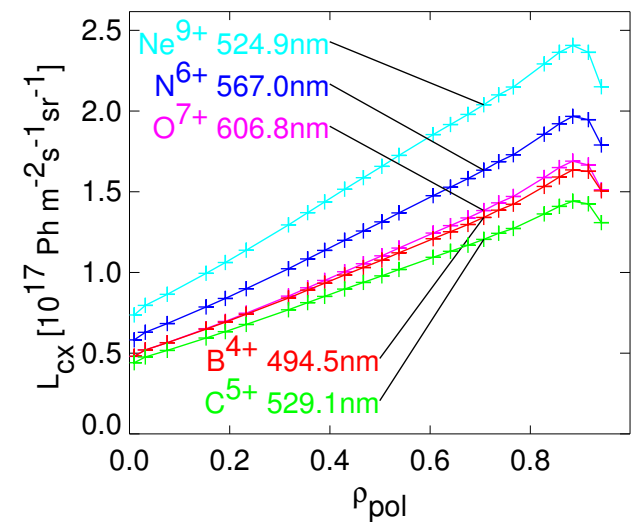

Figure 2. The expected radiance of the CX-lines from a number of light ions for equal impurity ion concentration of $1 \%$ for the core lines-of-sight.

Systematic errors due to the used emission rate coefficient might increase the relative uncertainty when comparing different ions. However, the variation of these rates is not so large as is demonstrated in Fig.2. Here, the expected radiance for an impurity ion concentration of $1 \%$ is shown for a number of CX-lines of the light elements from B to Ne using the plasma parameters as are shown below in Fig.7. The radiances differ by at most $40 \%$ between the different ions and the systematic error when comparing two ions is estimated to be at most $20 \%$. The absolute ion density can have further systematic errors due to the beam attenuation model, but comparison with the measured beam emission spectra showed that this error is not more than $15 \%[8,10]$.

In Fig.1, the density profile of the fully ionised oxygen is rather flat up to a certain radius close to the separatrix and then decays with a strong radial gradient towards the separatrix. The oxygen concentration is very low, about $2 \times 10^{-4}$, as is expected just after a fresh boronisation. The sum of the densities of $\mathrm{Ne}^{8+}$ and $\mathrm{O}^{8+}$ decreases towards the core where it remains at a constant density of about $9 \times 10^{15} \mathrm{~m}^{-3}$ for $\rho_{\text {pol }}<0.4$. This base level cannot come from $\mathrm{Ne}^{8+}$, since $T_{e}$, which is between 2.5 and $5 \mathrm{keV}$ in this range, is too high for He-like neon to exist. It must be caused by the fully ionised oxygen and the densities are in rather good agreement with the oxygen measurement from $\# 37061$, which are at a level of $1.3 \times 10^{16} \mathrm{~m}^{-3}$ in this range. For the next analysis step, the oxygen profile from \#37061 is scaled such that the mean value for $\rho_{\text {pol }}<0.4$ agrees with the densities of the measurement of $\mathrm{Ne}^{8+}$ and $\mathrm{O}^{8+}$ and the difference with 
this scaled oxygen density is then taken as the $\mathrm{Ne}^{8+}$ density. Since the oxygen level is so low, there are no large errors introduced by this method. In the outer radial region, the profile of $\mathrm{Ne}^{10+}$ is not as flat as $\mathrm{O}^{8+}$ and significantly decays towards the pedestal top. This is a consequence of the ionisation balance, since a considerable fraction of neon is still in the He-like stage and quite possibly also in the not measured H-like stage. The density of $\mathrm{Ne}^{8+}$ rises towards the edge and has about $2 / 3$ of the density of $\mathrm{Ne}^{10+}$ at the pedestal top where $T_{e}=600 \mathrm{eV}$ and $n_{e}=6 \times 10^{19} \mathrm{~m}^{-3}$.

\section{Modelling of the ionisation balance of neon}

The ionisation balance of neon has been modelled with the 1.5D-impurity transport code STRAHL [11]. STRAHL uses the radius $r=\sqrt{V / 2 \pi^{2} R_{a x i s}}$ as its coordinate, where $V$ is the volume enclosed by the flux surface. STRAHL solves the transport equations of all ion stages of an impurity where neighbouring ion stages are coupled via ionisation and recombination reactions. Thus, it accounts for the influence of the radial transport on the ionisation balance of an impurity. In addition to ionisation and recombination due to collisions with electrons, charge exchange from neutral deuterium atoms onto impurity ions will be shown to be another important recombination channel. The cross sections for this reaction were taken from [12] and are depicted in Fig.3.

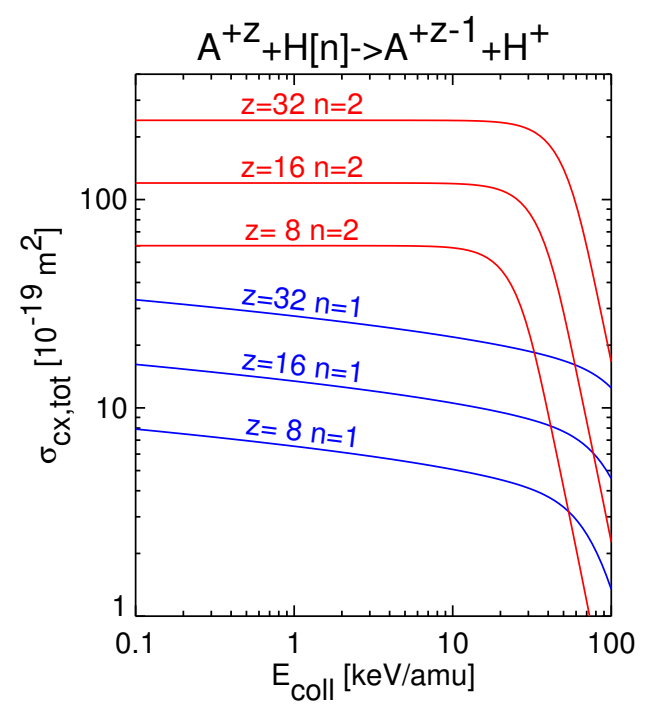

Figure 3. The cross sections for CX from hydrogen in the ground state $n=1$ and in the $n=2$-state with impurities with charge $Z=8,16$, and 32 as a function of the collision energy per atomic mass unit [12]. These cross sections are for CX into all levels of the receiving ion.

The blue lines in Fig. 3 show $\sigma_{c x}$ for CX from hydrogen in the ground state $n=1$ onto impurities with charges $Z=8,16$, and 32 versus the collision energy per atomic mass unit. The cross sections scale linearly with the impurity charge and are very weakly depending on the collision energy up to very high energies before they start to decay. 
The neutral beam heating at ASDEX Upgrade is operated up to an acceleration voltage of $93 \mathrm{keV}$ when using deuterium and thus, the collision energy is still in the flat region of the cross sections for $n=1$ at $E_{\text {coll }}=46.5 \mathrm{keV} / \mathrm{amu}$. The cross sections for hydrogen in $n=2$ are only about a factor of 10 larger than the $n=1$ cross sections. For typical fractions of excited hydrogen in $n=2$ of $\approx 0.5 \%$, the total CX-rates are therefore not much increased by the excited atoms. The reaction rate coefficients $\left\langle\sigma_{c x}\left(v_{n}-v_{i m p}\right)\right\rangle$ scale with the neutral velocity $v_{n}$ since $\sigma_{c x}$ depends so weakly on the collision energy and $v_{n}$ is usually much larger than $v_{i m p}$. Thus, the rate coefficients are larger by roughly a factor of $\sqrt{E_{\text {beam }} / k_{B} T_{n}}$ for beam neutrals with energy $E_{\text {beam }}$ than for thermal neutrals with temperature $T_{n}$.

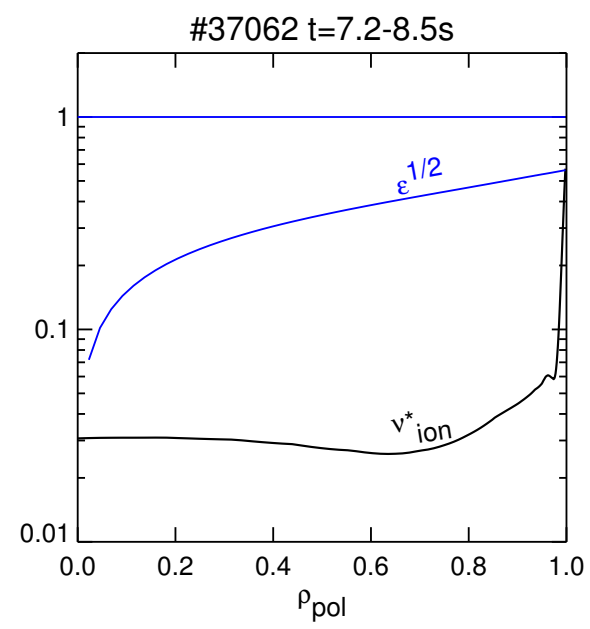

Figure 4. The normalised ionisation frequency $\nu_{i o n}^{*}$ of neon as defined in eq.(2) versus the poloidal flux label (see Fig.7 for the plasma parameters). It is below $\sqrt{\epsilon}$ everywhere, indicating that the ionisation balance for trapped and non-trapped ions is about constant on a flux surface even when the CX-reactions are not constant on the flux surface.

The neutral deuterium density from the neutral beam injection but also from the recycling thermal deuterium is certainly not constant on a flux surface in the confined plasma and it might be that the influence of the CX-reactions on the ionisation balance is either a local effect in the region of high neutral density or a global effect which affects the whole flux surface. We define a normalised ionisation frequency $\nu_{i o n}^{*}$ by multiplying the average ionisation rate $\nu_{i o n}$ with the characteristic transit time $\tau_{t}$ which a non-trapped impurity ion needs to travel the parallel distance $L=q R$ along the flux surface.

$$
\nu_{i o n}^{*}=\nu_{i o n} \tau_{t}=\nu_{i o n} \frac{q R}{v_{t h}}=\frac{\sum_{z} n_{z} \nu_{z}^{i o n}}{\sum_{z} n_{z}} q R \sqrt{\frac{m_{i m p}}{2 k_{B} T_{i}}}
$$

Here, we averaged the ionisation rates $\nu_{z}^{i o n}$ of all ion stages of neon by weighting with the density $n_{z}$ of the corresponding charge stage. When $\nu_{i o n}^{*}$ is much larger than 1 , a recombined ion will quickly re-ionise before travelling an appreciable stretch along the 
flux surface. Then, a local enhancement of the recombination rates only influences the ionisation balance in the vicinity of the disturbance. However, when $\nu_{i o n}^{*}$ is much less than 1 , a local change of the recombination rates changes the ionisation balance on the whole flux surface. This is the usual case in the presented discharges as can be seen in Fig.4. The normalised ionisation frequency is not only below 1, but also smaller than the square root of the inverse aspect ratio $\epsilon$, which shows that even the ionisation balance of trapped ions, whose parallel velocity is smaller by $\approx \sqrt{\epsilon}$, does not change on the flux surface. Thus, we assume for the following, that it is sufficient to take only the flux surface averages of the neutral densities and the corresponding CX-rates into account and that a $1.5 \mathrm{D}$ transport code is a good approximation for the confined region.

In the region around the $\mathrm{X}$-point the field lines are almost purely toroidal and the connection length $L_{c o n}$ to the outboard equator is especially long. When using a stricter $\mathrm{x}$-point version of the normalised ionisation frequency $\nu_{i o n}^{*, x p t}=\nu_{\text {ion }} L_{\text {con }} / v_{t h}$, it is found that $\nu_{i o n}^{* x p t}$ of neon becomes larger than 1 for $\rho_{\text {pol }}=0.994$ for the equilibrium of Fig.4. This flux surface has a radial distance $\Delta r_{s e p}=0.7 \mathrm{~cm}$ to the separatrix and a vertical distance of $\Delta z_{x p t}=10 \mathrm{~cm}$ above the lower X-point of this lower single null plasma. Thus, a large neutral density extending up to a few $\mathrm{cm}$ above the X-point will lead to a local change of the ionisation equilibrium. These neutrals will not be included in our flux surface average and a calculation of the ionisation equilibrium in this area would require a $2 \mathrm{D}$ model.

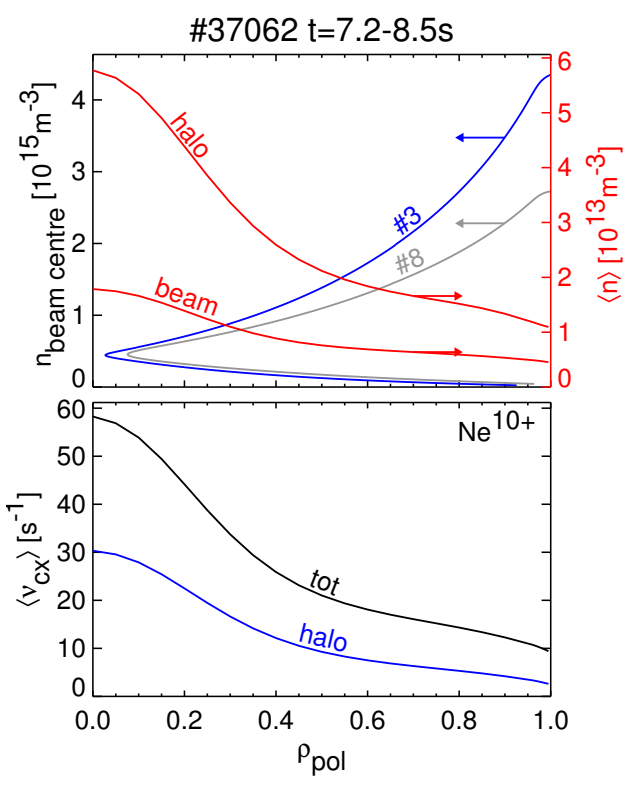

Figure 5. Beam and halo densities for \# 37062 at $\mathrm{t}=7.2-8.5 \mathrm{~s}$ from the two NBI sources (see Fig.7 for the plasma parameters) and the corresponding CX-rates for $\mathrm{Ne}^{10+}$.

The CX-rates due to the neutral beams are prepared by calculating the beam attenuation with the same code as used for the evaluation of the CXRS measurements [8]. The resulting beam density profiles just have to be averaged over the corresponding 
flux surfaces and multiplied with the rate coefficients for CX-collisions to obtain the required flux surface averages. Fig.5 shows the result of this calculation for $t=7.2-8.5 \mathrm{~s}$ from \#37062. Here, the active beams \#3 and \#8 with $60 \mathrm{keV}$ and $93 \mathrm{keV}$ acceleration voltage at an injection power of $2.5 \mathrm{MW}$ each are depicted. The upper graph shows the evolution of the neutral beam density in the centre of each beam versus $\rho_{\text {pol }}$. The red curves (right y-axis) give the total flux surface averaged neutral density of the beams and of the thermal halo neutrals. Both curves are peaked in the centre. Even though the halo densities dominate, they only contribute about $50 \%$ to the charge exchange rate. This is displayed in the bottom graph, where the total CX-rate of $\mathrm{Ne}^{10+}$ and the part caused by the halo neutrals is shown.

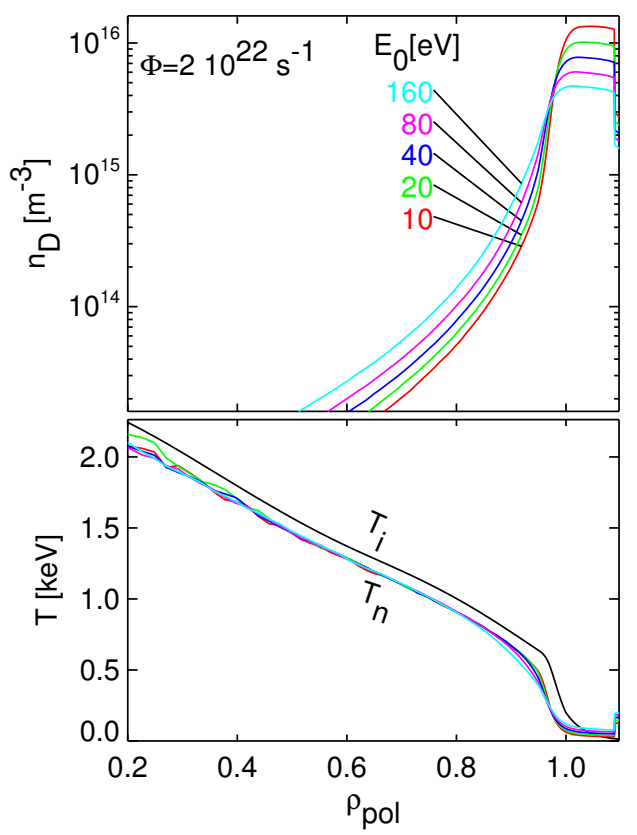

Figure 6. Radial profiles of the neutral density and the temperature of the recycling deuterium from a 1D Monte Carlo code for \# 37062 at $t=7.2-8.5 \mathrm{~s}$ (see Fig.7 for the plasma parameters).

It will be shown below, that the recycling deuterium is by far the main contributor to the CX-reactions which are needed to explain the ionisation balance of neon in the pedestal region and its neutral density will be determined by a fit procedure. However, STRAHL needs the density and the temperature of the neutrals over the whole plasma radius. The real neutral density is a complex three dimensional function. For our purpose, just the flux surface averaged neutral density is important as has been discussed above. We used a simple one dimensional Monte Carlo code to calculate a class of possible radial profiles, which just depend on two parameters: the source rate of neutral atoms $\Phi$ and the energy $E_{0}$, with which a neutral starts at the wall. In the Monte Carlo code the same cylindrical geometry as in STRAHL is used. A neutral D atom starts at the radius of the main chamber limiters with purely radial velocity. It can either be ionised due to electron impact or it can undergo a CX-collision with a deuterium ion. 
After a CX-collision the newly created neutral atom will have a random velocity out of the Maxwell distribution corresponding to the ion temperature at this location. Now the code follows this new atom and eventually all subsequent daughter atoms until they are either finally ionised by electron impact or move out of the radial grid. A few million markers are followed to obtain a radial profile. The neutral density $n_{D}$ is proportional to the time the markers spend in a certain radial bin and a neutral temperature $T_{n}$ is obtained by multiplying the time with the kinetic energy of the marker to determine the mean kinetic energy of all markers in the bin.

A number of profiles for the plasma parameters from \#37062 at $t=7.2-8.5 \mathrm{~s}$ are shown in Fig.6. The same source rate of $\Phi=2 \times 10^{22} \mathrm{~s}^{-1}$ is used for all density profiles in the upper graph but the initial energy $E_{0}$ of the atom varies between 10 and $160 \mathrm{eV}$. The initial energies in ASDEX Upgrade with tungsten as plasma facing material are high since about $70 \%$ of the $\mathrm{D}$ ions impinging on $\mathrm{W}$ surfaces are reflected as neutral atoms with an energy of about $70 \%$ of the energy of the impinging ion [13]. Thus, when using a simple estimate for the energy $E_{i n c}$ of the impinging ion after acceleration in the sheath, i.e. $E_{i n c}=5 k_{B} T$ for $T=T_{e}=T_{i}$, the $E_{0}$-grid used in Fig. 6 corresponds to a grid of temperatures at the wall of $2.8-46 \mathrm{eV}$.

The density profiles become a bit wider with increasing $E_{0}$. We use the radius at which the density decays to $20 \%$ of the density at the separatrix to quantify this and the difference of this radius to the radius of the separatrix $\Delta r_{20}$ rises from $3.6 \mathrm{~cm}$ to $9.4 \mathrm{~cm}$. It scales as $\Delta r_{20}=3.6 \mathrm{~cm}\left(E_{0} / 10 \mathrm{eV}\right)^{0.34}$. When the electron density at the pedestal is slightly higher as in \#37062 at $t=5.2-6.4 \mathrm{~s}$ (see Fig.9) the scaling with $E_{0}$ becomes even weaker $\Delta r_{20}=3.4 \mathrm{~cm}\left(E_{0} / 10 \mathrm{eV}\right)^{0.30}$. The mean dwell time $\tau_{n}$ of a neutral in the plasma is a few microseconds and decreases with increasing energy since a higher fraction of the atoms reside in regions with higher electron density and temperature. It scales as $\tau_{n}=3.6 \mu \mathrm{s}\left(E_{0} / 10 \mathrm{eV}\right)^{-0.28}$. The edge losses contribute $40 \%$ to the total loss of the neutrals at $10 \mathrm{eV}$ and $32 \%$ at the highest energy. The temperature profiles of the neutrals in the lower graph of Fig.6 are almost identical for all starting energies and always a bit shifted to the inside with respect to the ion temperature. This reflects that most of the neutrals at a certain radius stem from a CX-reaction at a larger radius where $T_{i}$ is lower.

A first STRAHL modelling of the neon balance was done without using CXreactions. Here, only the electron density $n_{e}$ and the electron temperature $T_{e}$ are needed to compute the ionisation and recombination rates. The temporally averaged profiles for \#37062 at $t=7.2-8.5 \mathrm{~s}$ are shown in the upper left and lower left box of Fig.7. We just use temporally averaged transport coefficients, i.e. without resolving the transport invoked by ELMs, to model the average density profiles of neon and each simulation is run until a temporal equilibrium is achieved. The diffusion coefficient $D$ is displayed in the lower right box. In the radial range from $\rho_{p o l}=0.2-0.95$ it is proportional to the effective heat diffusivity $\chi_{\text {eff }}$. It will be shown below that the proportionality factor can be determined from the $\mathrm{Ne}^{8+}$ profile when CX-reactions are included in the model. Here, the result does not strongly depend on its value, but is set to the same value to achieve 

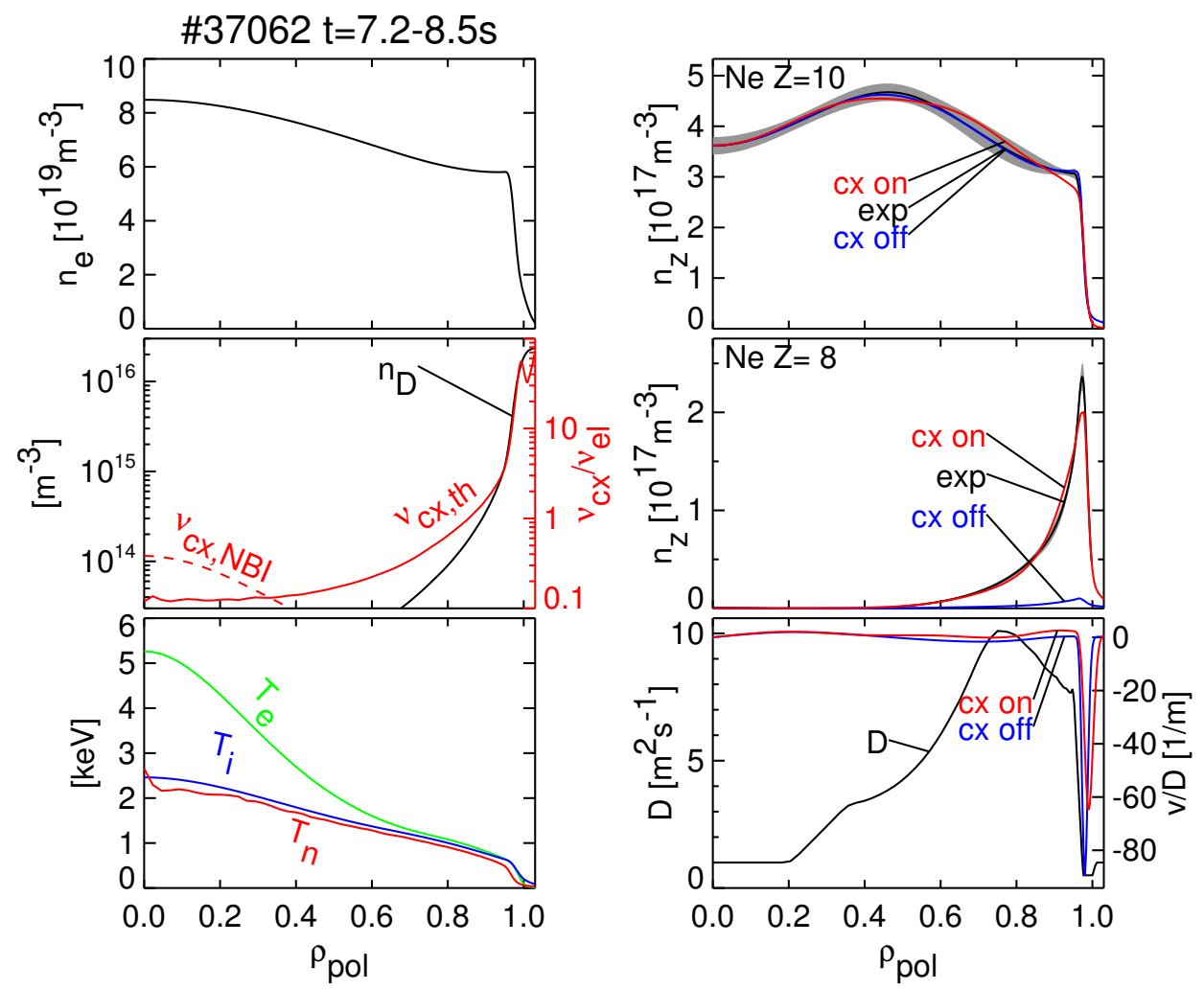

Figure 7. Radial profiles of the plasma parameters and the fit of the measured neon density profiles with and without the inclusion of CX-reactions for \#37062 at $t=7.2$ 8.5 s. The large He-like neon density can only be explained when including the CXreactions with neutral deuterium using the profiles of the neutral deuterium density as shown in the middle left box.

a consistent comparison. In the region of the H-mode edge transport barrier (ETB), $D$ is set to drop to a value of $0.5 \mathrm{~m}^{2} / \mathrm{s}$ and rises again to a higher value in the SOL. In the centre, it has a constant value of $1 \mathrm{~m}^{2} / \mathrm{s}$. The fit procedure has the parameters, which describe the profile of the drift velocity $v$, as free parameters. The ratio $v / D$ determines the normalised gradient of the total neon density: $\left(1 / n_{t o t}\right)\left(d n_{t o t} / d r\right)=v / D$. The parametrisation of $v / D$ uses the sum of a spline function with knots at $\rho_{p o l}=0.2,0.4$, 0.6, 0.8, and 0.9 and a Gaussian function in the ETB with peak value, peak position, and width as parameters. The neon density of all charge stages and at all radii is proportional to the source rate. It is adjusted such as to fit the sum of the densities of the He-like and fully ionised ion at $\rho_{\text {pol }}=0.9$. The best fitting profile of $v / D$ is displayed in the lower right box of Fig.7 and the comparison of modelled (blue lines with label cx off) and experimental neon densities (black lines with label exp) is shown in the upper right box for $Z=10$ and in the middle box for $Z=8$. The fully ionised species can be fitted rather well, where the large inward drift in the transport barrier is needed to describe the strong gradient in this region. However, the density of the He-like stage is much lower in the model than in the experiment. It is too low by a factor of between 
15 and 28 in the range from $\rho_{p o l}=0.9-0.98$.

This large discrepancy can only be diminished when allowing for higher recombination rates, which can be provided by the CX-reactions. The CX-reactions induced by beam neutrals and recycling neutrals are now included. Here, we also need the ion temperature $T_{i}$ and the neutral temperature $T_{n}$ (see lower left box) for calculation of the CX-rates with the recycling neutrals, which depend on the reduced temperature $T_{\text {red }}=\left(T_{i} m_{D}+T_{n} m_{N e}\right) /\left(m_{D}+m_{N e}\right)$. The fit procedure has three additional parameters: the source rate and starting energy $E_{0}$ of the recycling neutrals and the proportionality factor $f_{\chi}$ which determines the diffusion coefficient $D=f_{\chi} \chi_{e f f}$. Here, $E_{0}$ is bound to only vary within the limits shown in Fig.6 such that the neutral profile can not become arbitrarily wide. The fitted model profiles of the densities and $v / D$ are shown with red lines in the right boxes, while the corresponding diffusion coefficient $D$ is shown with a black line. The neutral density is shown in the left middle box. The density of the He-like neon can now be much better explained, even though there is no perfect agreement between model and experiment within the error band of the measurement.
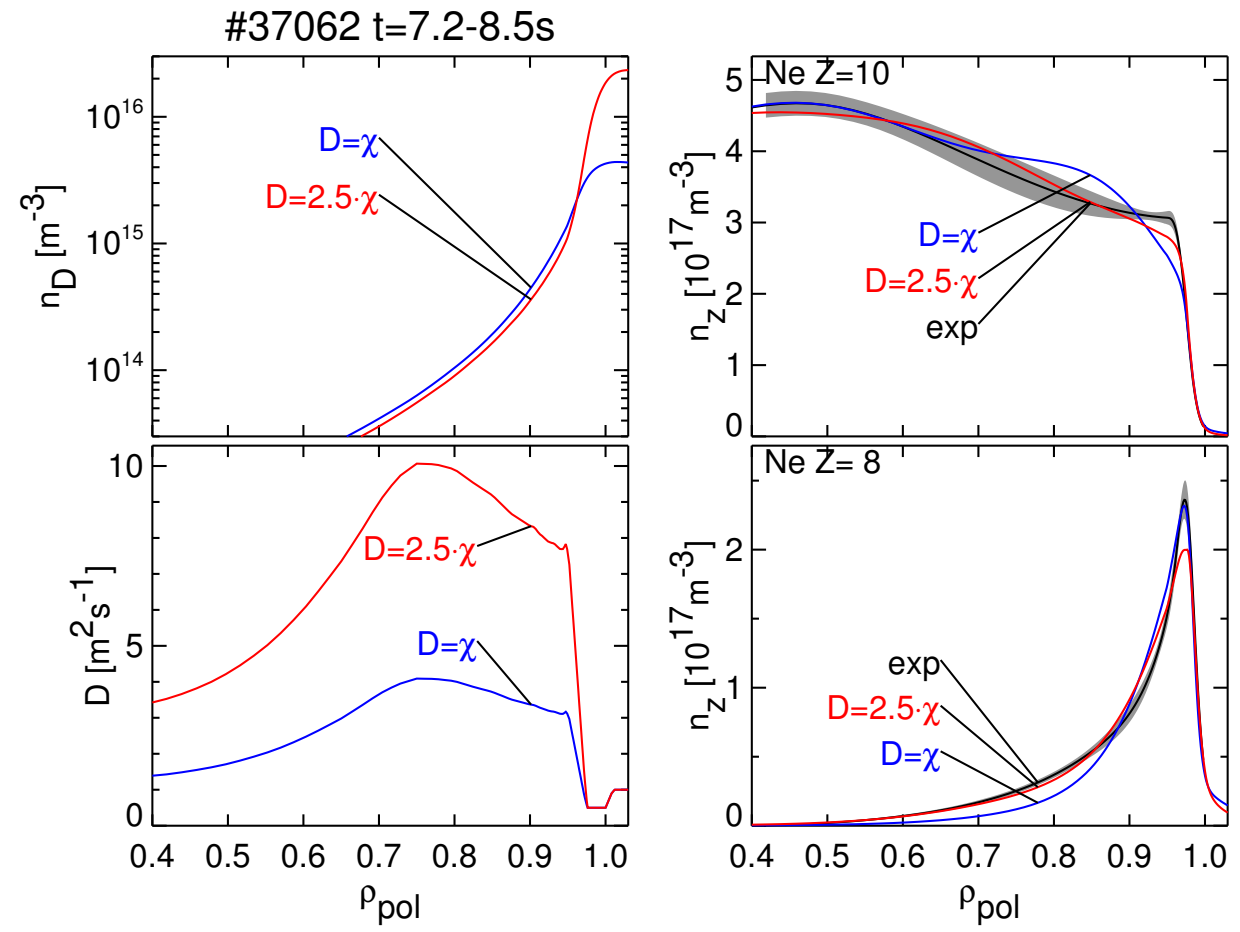

Figure 8. Radial profiles of $n_{D}$ and the fit of the measured neon density profiles with two settings for the diffusion coefficient for \#37062 at $t=5.2-6.4 \mathrm{~s}$. The long decay length of the He-like neon density can only be explained with the higher diffusion coefficient.

The fit yields for the neutral density at the separatrix $n_{D, s e p}=1.9 \times 10^{16} \mathrm{~m}^{-3}$ and $n_{D}=4.1 \times 10^{15} \mathrm{~m}^{-3}$ at the maximum position of the He-like neon density, which is further inward by $\Delta r_{s e p}=r_{s e p}-r=3.6 \mathrm{~cm}$. The shape of the $n_{D}$ profile corresponds to an initial deuterium energy of $E_{0}=10 \mathrm{eV}\left(\Delta r_{20}=3.6 \mathrm{~cm}\right)$. These densities lead to a strong increase 
of the recombination rates. This can be quantified by forming the averages of the recombination rates over the charge stages of neon, which were already introduced for the ionisation rates in eq.(2) and by dividing the rate for charge exchange recombination with the rate for electronic recombination. These ratios are depicted in the middle left box of Fig.7 with a dashed red line for the beam neutrals and a solid red line for the recycling neutrals. The effect of the beam neutrals can not be judged by comparing to the measurements since the He-like ion does not exist in the central part of the plasma. The recycling neutrals cause more recombinations than the electrons, i.e. the ratio is larger than 1 , for $\rho_{\text {pol }}>0.85$, where $n_{D}=1.7 \times 10^{14} \mathrm{~m}^{-3}$ and $n_{D} / n_{e}=2.8 \times 10^{-6}$. The ratio reaches a value of 25 at $\rho_{\text {pol }}=0.98\left(\Delta r_{\text {sep }}=2.3 \mathrm{~cm}\right)$ with $n_{D}=8.5 \times 10^{15} \mathrm{~m}^{-3}$ and $n_{D} / n_{e}=2.7 \times 10^{-4}$ and a bit further out it has a maximum value of 50 at $\Delta r_{\text {sep }}=1.1 \mathrm{~cm}$ with $n_{D} / n_{e}=7.2 \times 10^{-4}$.

For $f_{\chi}$, the fit obtains a value of 2.5. It is mainly determined by the decay of the He-like density, while inside of $\rho_{\text {pol }}=0.4$, where the density of He-like neon is zero, the fits are insensitive to the setting of $D$ in this region. Fig. 8 compares the best fit with CX-reactions with the case where the diffusion coefficient was set to $D=\chi_{\text {eff }}$. The decay length of the He-like density is too low with the low diffusion coefficient. The fit procedure increases the inward pinch $v / D$ and produces a local maximum of the density of $Z=10$ around 0.85 outside the error bar (grey region), but still the He-like density is too low in this region.

The same fitting with and without CX-reactions has also been performed for the time range $t=5.2-6.4 \mathrm{~s}$ of $\# 37062$. Here, the deuterium puff level was increased from $5.0 \times 10^{21} \mathrm{~s}^{-1}$ by about a factor of 5 to $2.6 \times 10^{22} \mathrm{~s}^{-1}$. An increase of $n_{D}$ inside the separatrix and consequently a larger impact of CX-reactions might be expected. At the pedestal top, the higher puff level leads to a modest increase of $n_{e}$ from $5.8 \times 10^{19} \mathrm{~m}^{-3}$ to $6.8 \times 10^{19} \mathrm{~m}^{-3}$ and a decrease of $T_{e}$ from $600 \mathrm{eV}$ to $500 \mathrm{eV}$. However, the densities of both neon stages are a factor of 2.1 lower when increasing the D puff level, which is partly due to an increase of the effective pumping speed with higher divertor density [14] and partly due to an increase of the ELM frequency from $150 \mathrm{~Hz}$ to $310 \mathrm{~Hz}$, which reduces the time averaged inward pinch in the ETB $[15,16]$. Actually, the fits (including the CX-reactions) deliver for the inward pinch in the pedestal a reduction of the peak from $v / D=-67 \dot{\mathrm{m}}^{-1}$ to $v / D=-46 \dot{\mathrm{m}}^{-1}$. Again, the model without CX-reactions fails to describe the He-like neon densities, which are too low by a factor between 8 and 12 for $\rho_{\text {pol }}>0.9$, i.e. not as large as in the low puff case. These lower factors are caused by the lower electron temperature, which already shifts the ionisation balance a bit towards lower ion stages, but by far not strong enough to fit the measurement. The model with CX-reactions leads to a good fit of the He-like neon densities. The fitted deuterium density profile at the separatrix is similar to the previous case with a lower separatrix value of $n_{D, s e p}=1.3 \times 10^{16} \mathrm{~m}^{-3}$, but a higher initial deuterium energy of $E_{0}=23 \mathrm{eV}\left(\Delta r_{20}=4.4 \mathrm{~cm}\right)$. The needed increase of the electronic recombination rates is not as high as in the time range with the low D-puff and almost the same deuterium density delivers this lower increase. At $\rho_{\text {pol }}=0.95, n_{D}$ is $1.1 \times 10^{15} \mathrm{~m}^{-3}$ in the low puff 

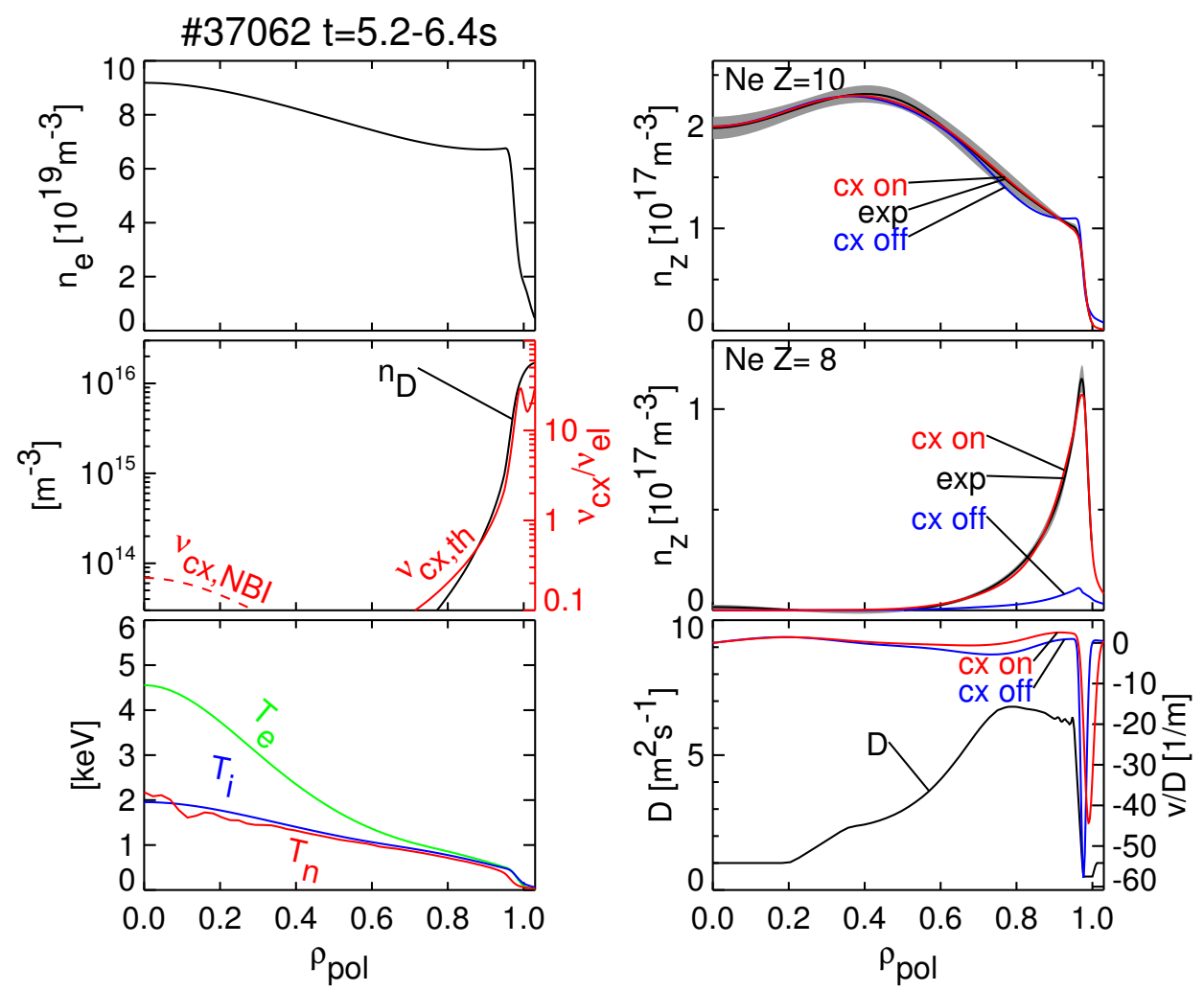

Figure 9. Radial profiles of the plasma parameters and the fit of the measured neon density profiles with and without the inclusion of CX-reactions for \#37062 at $t=5.2$ $6.4 \mathrm{~s}$. The large He-like neon density can only be explained when including the CXreactions with neutral deuterium using the profiles of the neutral deuterium density as shown in the middle left box.

case and $1.0 \times 10^{15} \mathrm{~m}^{-3}$ in the high puff case. The increase of the puff level certainly leads to a higher recycling flux, however, the neutrals do not seem to penetrate so well into the confined region.

The diffusion coefficient is more or less constant for $\rho_{\text {pol }}>0.75$ up to the edge transport barrier and does not show this decrease as in the low puff case. This change is due to the lower radiated power density at the plasma edge in the high puff case since the neon density is lower. The lower radiated power keeps the conducted power at larger radii at a higher level, which is then reflected in a larger $\chi_{e f f}$ at these radii. The fit yields for $f_{\chi}$ a value of 1.7 .

\section{Influence on the main chamber neon radiation}

The much higher density of partially ionised neon inside of the pedestal top leads to an increase of the total neon radiation, which is much larger than expected without CXreactions, since line radiation from the measured He-like stage, but also from $\mathrm{H}$-like and Li-like ions can radiate much more compared to the bremsstrahlung and recombination radiation invoked by the fully ionised stage. This is certainly the most relevant effect of 
the changed ionisation balance. For the two fits with and without CX-reactions shown in Fig.7, the corresponding radial emissivity profiles are shown in the left box of Fig.10.
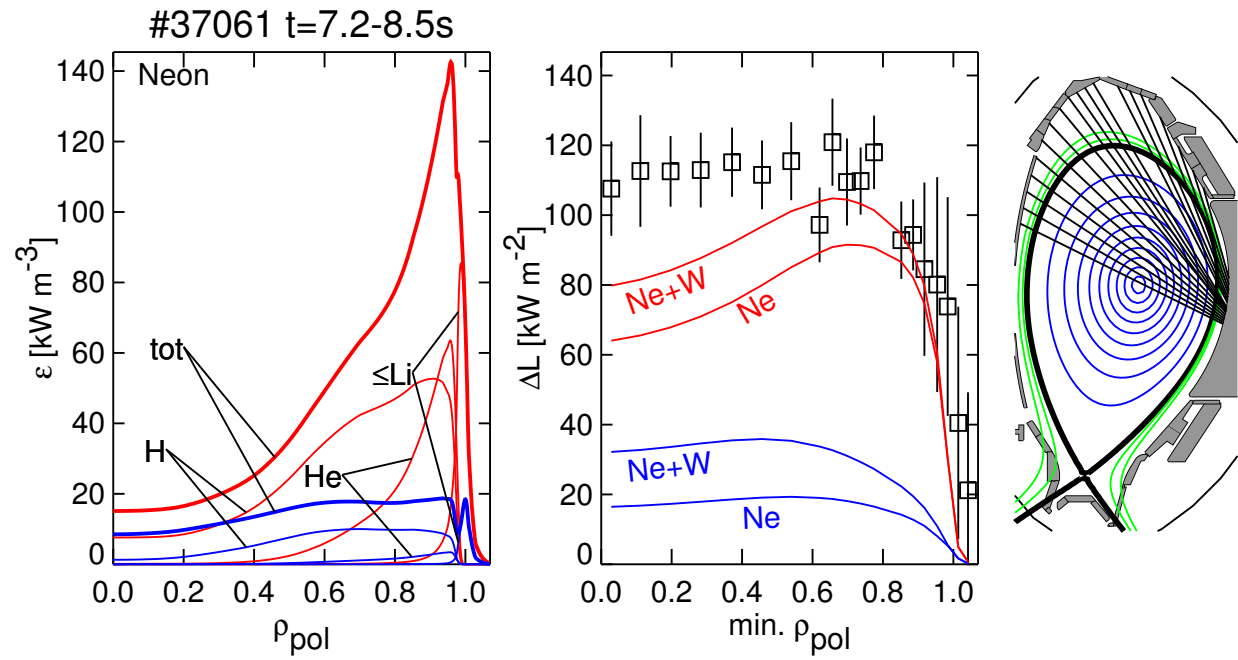

Figure 10. Left box: radial profiles of the neon emissivity due to line radiation of H-like, He-like, all charges stages $\leq$ Li-like, and the total neon emissivity including bremsstrahlung and continuum radiation for the fit from Fig.7 with (red) and without (blue) CX-reactions. Right box: measured changes of the radiances of a main chamber bolometer camera from $t=7.2-8.5 \mathrm{~s}$ to before the neon puff and modelled radiances of the two neon-fits and with inclusion of $\mathrm{W}$ radiation due to a change of the $\mathrm{W}$ concentration by $\Delta c_{W}=9.5 \times 10^{-6}$.

In the left plot of Fig.10, the emissivity profiles of the line radiation of $\mathrm{Ne}^{9+}(\mathrm{H}-$ like), $\mathrm{Ne}^{8+}$ (He-like) and of all stages with charges $Z \leq 7$ ( $\leq$ Li-like) are plotted together with the total radiation of neon including the bremsstrahlung and the recombination radiation. Red lines are for the case with CX and blue lines for the case without CX. The line radiation from $\mathrm{Ne}^{9+}$ extends up to the centre of the plasma and the total radiated power within the separatrix $P_{\text {rad,sep }}$ from this contribution increases by a factor of 9.6 when considering the CX-recombination. $P_{\text {rad,sep }}$ from line radiation of the He-like stage is stronger by a factor of 16 and for the charge stages below $\mathrm{Ne}^{8+}$, the factor is 4.8 . These lower charge stages contribute about $45 \%$ to the total $P_{\text {rad,sep }}$ of neon in both cases and since the continuum radiation does not change so strongly, the effect of CX is to cause an increase of $P_{\text {rad,sep }}(\mathrm{Ne})$ by a factor of 5 from $220 \mathrm{~kW}$ to $1.1 \mathrm{MW}$.

Unfortunately, there are no measured profiles of the radiances emitted by He-like and H-like resonance lines, which would yield a more straight forward comparison with the model. However, the total radiation in the main chamber was also about a factor of 2 higher in the time range $t=7.2-8.5 \mathrm{~s}$ than just before the neon puff. Thus, the difference of the radiances with a phase just before the neon injection will be compared with the fit-results. The comparison is made for \#37061, since in \#37062, there is already some recycling neon present before the start of the neon puff. The electron density before the puff and in the phase around $t=8 \mathrm{~s}$ is almost the same. A set of 
lines-of-sight that is the least influenced by divertor radiation is chosen. The changes of the radiances $\Delta L$ of the upper lines-of-sight of a main chamber bolometer camera are shown in the right box of Fig.10 and next to the box the geometry of the lines-ofsight. The lines with the label Ne yield the radiances from the neon radiation of the two fits with (red) and without (blue) CX-reactions. The model with CX is much closer to the measurement. The tungsten concentration, as measured by VUV-spectroscopy, is also higher in the time range with neon by $\Delta c_{W}=9.5 \times 10^{-6}$ and the lines with the label $\mathrm{Ne}+\mathrm{W}$ include this change of the $\mathrm{W}$-radiation when using a flat radial profile for $c_{W}$. The fit is not perfect and other impurities, which have not been monitored, might also have changed somewhat. However, the main conclusion is unaffected: The large change of the radiation from the confined plasma can only be explained with an ionisation balance that is shifted away from the fully ionised species towards lower ion stages, which can emit line radiation.

\section{Application to an H-mode plasma with strong argon radiation}

In the type-I ELMy H-mode discharge $\# 36448$ with $I_{p}=0.8 \mathrm{MA}$, toroidal field $B_{t}=2.5 \mathrm{~T}$, heating power $P=12.4 \mathrm{MW}$, and line averaged density of $9.2 \times 10^{19} \mathrm{~m}^{-3}$ a strong argon puff was applied to drive the power crossing the separatrix down towards the H-L-limit with the ultimate goal of ELM stabilisation [17, 14]. Even though reduced-size ELMs remained in this discharge, the detected radiances by bolometers, soft X-ray cameras and by the SPRED spectrometer measuring in the vacuum ultraviolet (VUV) range are strongly dominated by argon radiation, such that this plasma is a good test case to study the effect of CX-recombination on the pedestal radiation of argon.

In Fig.11, average profiles for the time range 4.2-4.4 $\mathrm{s}$ are shown. At this time, the highest heating and radiated powers are achieved just before the appearance of neoclassical tearing modes due to the high plasma pressure (see [14] for details). The two uppermost plots on the left display $n_{e}, T_{e}$ and $T_{i}$.

The density of argon was inferred from the soft X-ray radiation. In the upper right box, the soft X-ray (SXR) emissivity profile from tomographic inversion of the measured radiances [18] is shown as a grey band which reflects the assumed relative uncertainty of 10\%. The soft X-ray cameras have a $75 \mu \mathrm{m}$ thick Be-filter in front of the silicon diodes. The quantum efficiency of the setup is calculated with the transmission and absorption data from [19] and a model for the diode response from [20]. It is larger than $1 \%$ for photon energies above $1.25 \mathrm{keV}$. Thus, line radiation from $\mathrm{H}$-like and He-like argon is detected. The modelled soft X-ray radiation adds up all lines from an ion multiplied with the corresponding quantum efficiency of the camera. Bremsstrahlung and freebound transitions from radiative recombination are added using energies of hydrogen-like states with the appropriate Gaunt factors [21], which also depend on the photon energy. The line radiation emitted by the radiatively recombined ion is also included. For dielectronic recombination, the prescription of [22] is applied using excitation energies and oscillator strengths of two effective excited states. Here, the radiative decay of 


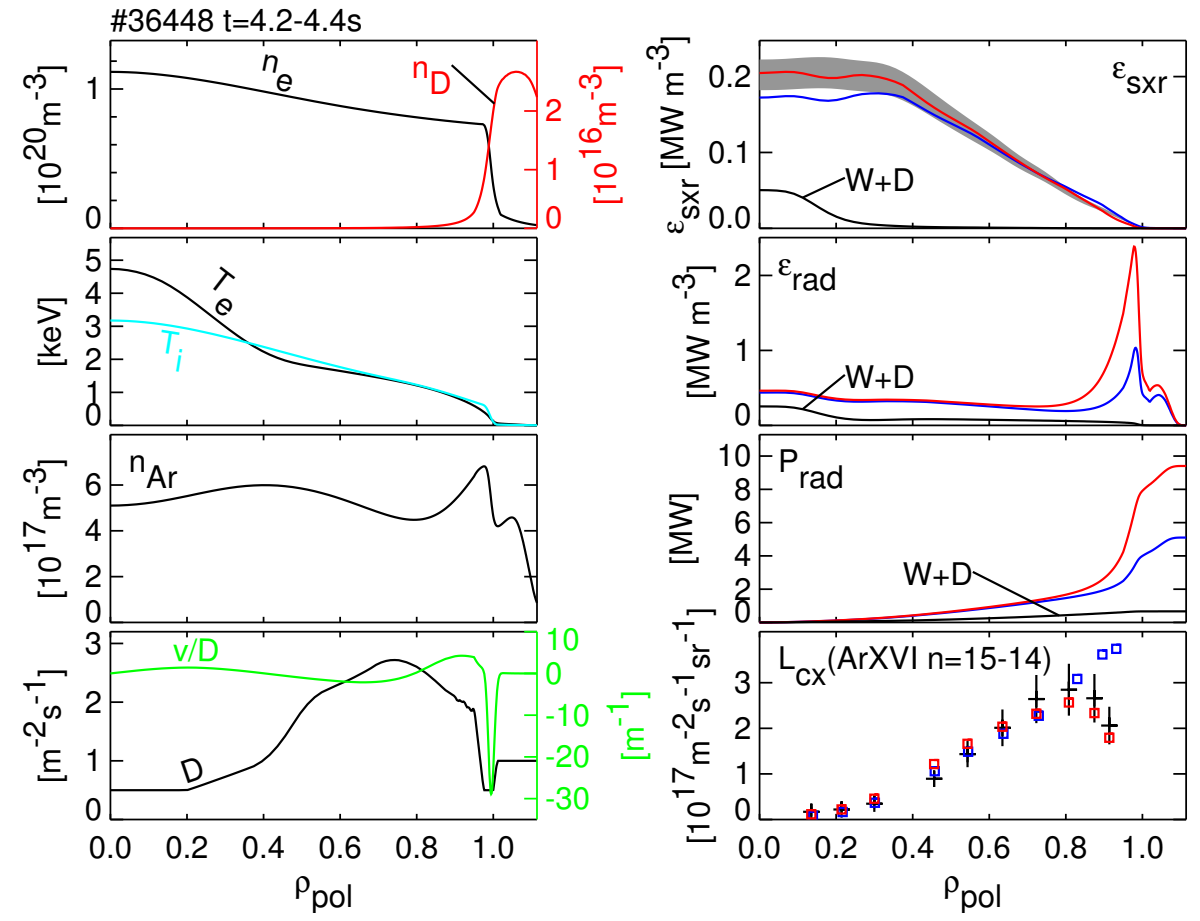

Figure 11. Radial profiles of the plasma parameters and the fitted argon density profiles from the soft X-ray emission. Comparison of the calculated total emission, $P_{\text {rad }}$ and the radiance of the $n=15$-14-transition of ArXVI measured with CXRS with (red) and without (blue) the inclusion of CX-reactions for \#36448 at $t=4.2-4.4 \mathrm{~s}$. The total radiation in the pedestal region strongly increases when including CX-reactions with neutral deuterium using the profiles of the neutral deuterium density as shown in the upper left box.

the excited electron is included, while the radiation from the recombined electron is approximated to have a photon energy equal to the ionisation energy of the recombined ion.

The tungsten concentration $c_{W}$ in the confined plasma has been deduced from $\mathrm{W}$-spectra in the VUV region measured with a grazing incidence spectrometer. The quasi-continuum at $5 \mathrm{~nm}$ emitted by ions around $\mathrm{W}^{30+}$ yields $c_{W}=2 \times 10^{-5}$ at a radial location with $T_{e} \approx 1.5 \mathrm{keV}$, while line emission from $\mathrm{W}^{40+}-\mathrm{W}^{45+}$ gives a central concentration of $c_{W}=1 \times 10^{-4}$ [23]. This rather large central W-concentration causes central radiation which needs to be taken into account for the analysis of bolometric and SXR measurements. W was therefore included in the analysis with STRAHL. The drift parameter and the influx for $\mathrm{W}$ were adjusted such that that the measured concentrations are matched, i.e. the edge value for the radial region outside of $\rho_{\text {pol }}=0.3$, while inside there is a rise of $c_{W}$ to the central value. The chosen radial shape of the $\mathrm{W}$-concentration is reflected in the SXR and total emission of $\mathrm{W}$ which is shown in the upper right plots of Fig.11. Here, the label $\mathrm{W}+\mathrm{D}$ indicates, that also the small contribution of bremsstrahlung from Coulomb collisions with the main ion was added to the $\mathrm{W}$ radiation. For $\mathrm{W}$, the $\mathrm{CX}$-recombination rates are too small to cause significant 
changes of the ionisation balance.

There is also information on the density profile of $\mathrm{Ar}^{16+}$ from CXRS, which measured the $n=15$-14-transition of ArXVI at 541.2nm, however, calculated emission rate coefficients for this line are not in agreement with the experiment by a factor on the order of 3 (see [24] for details). Thus, we scaled the coefficients to fit to the soft $\mathrm{X}$-ray data and have just an information on the shape of the $\mathrm{Ar}^{16+}$ profile from this measurement.

The fitting method is very similar as was used for neon. Here, we first fit the case with inclusion of the CX reactions. Again, we use a spline plus a Gaussian peak in the ETB region to parametrise $v / D$. The profile of $v / D$ in the bulk of the plasma inside of the ETB is determined by the profile of the SXR emissivity. The lowest box in the left column of Fig.11 shows the $v / D$-profile and in the second lowest box the density profile of argon is displayed, which is rather flat in the bulk of the plasma. The radiances of the VUV-lines (see discussion of Fig.14) are sensitive to the peak of $v / D$ in the ETB. The neutral density profile has a constant shape with $\Delta r_{20}=4.3 \mathrm{~cm}$ and its height is determined by the total radiation emitted in the pedestal region to obtain agreement with the bolometric data from the main plasma (see Fig.13). A good fit was obtained with $n_{D, s e p}=2 \times 10^{16} \mathrm{~m}^{-3}$. The diffusion coefficient influences a bit the modelled profile of ArXVI but the dependence is rather weak and $f_{\chi}=1$ was used. The STRAHL model was then invoked with the same transport coefficients without using the CX recombination.

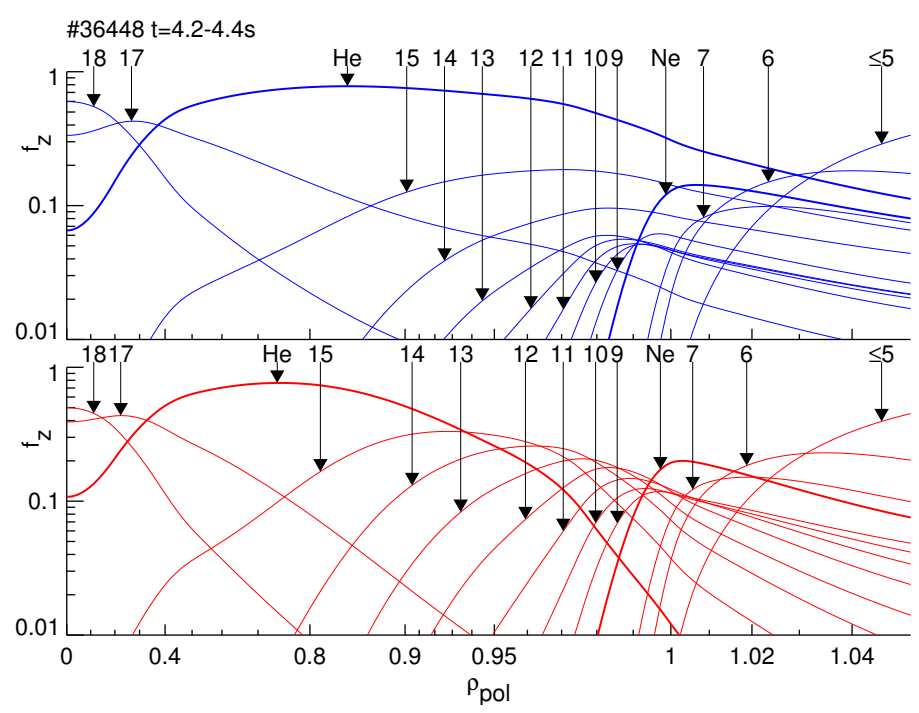

Figure 12. The radial profiles of the fractional abundance of the ion stages of argon without (blue) and with (red) CX recombination for \#36448 $t=4.2-4.4 \mathrm{~s}$.

The modelling results with CX are shown with red lines and squares in the right column, and with blue colour for the case without CX. The SXR emissivity is of course fitted by the model and defines the Ar density. When switching off CX recombination, the central SXR emission drops slightly since the ionisation balance is shifted towards 
the fully ionised argon and reduces the line emission of Ar in the centre. Here, we see the effect of the NBI neutrals on the ionisation balance. At $\rho_{\text {pol }}=0.9$ on the other hand, the SXR emission without CX is slightly increased due to a stronger abundance of Helike Ar. The difference between the two cases is much larger for the total emissivity and the total radiation emitted within a certain radius (second and third plot on the right). The CX recombination causes a wider and higher radiation peak in the pedestal. The total radiation inside the separatrix increases from $P_{\text {rad sep }}=4.0 \mathrm{MW}$ without CX to $P_{\text {rad }, s e p}=7.9 \mathrm{MW}$ with CX. Since $\mathrm{W}$ and D cause $P_{\text {rad,sep }}=0.66 \mathrm{MW}$, the radiation of only argon is increased by a factor of 2.2. Finally, we also see a small effect on the CXRS radiances measured on the outer lines-of-sight, where the CX-off points are above the measurement.

The change of the ionisation balance invoked by the CX-recombination is shown in Fig.12. The fractional abundance $f_{z}=n_{z} / n_{\mathrm{Ar}}$ without CX is shown in the upper graph and with $\mathrm{CX}$ in the lower graph. The ion stages with charges $Z \leq 5$ are combined in one line. The horizontal axis is non-linear to have increased resolution in the pedestal region. There is a small shift from fully ionised to partially ionised stages in the centre of the plasma due to the CX recombination with NBI neutrals, which explains the lower soft X-ray radiation in the centre without CX (see Fig.11) because less line radiation from the H-like stage is emitted. In the pedestal and in the near SOL, the He-like stage has highest fractional abundance without CX. This is strongly changed when CX is on. Here, the stages with $Z=8-15$ have higher abundances than the He-like stage and can contribute more efficiently to the total emission of Ar in this part of the plasma.
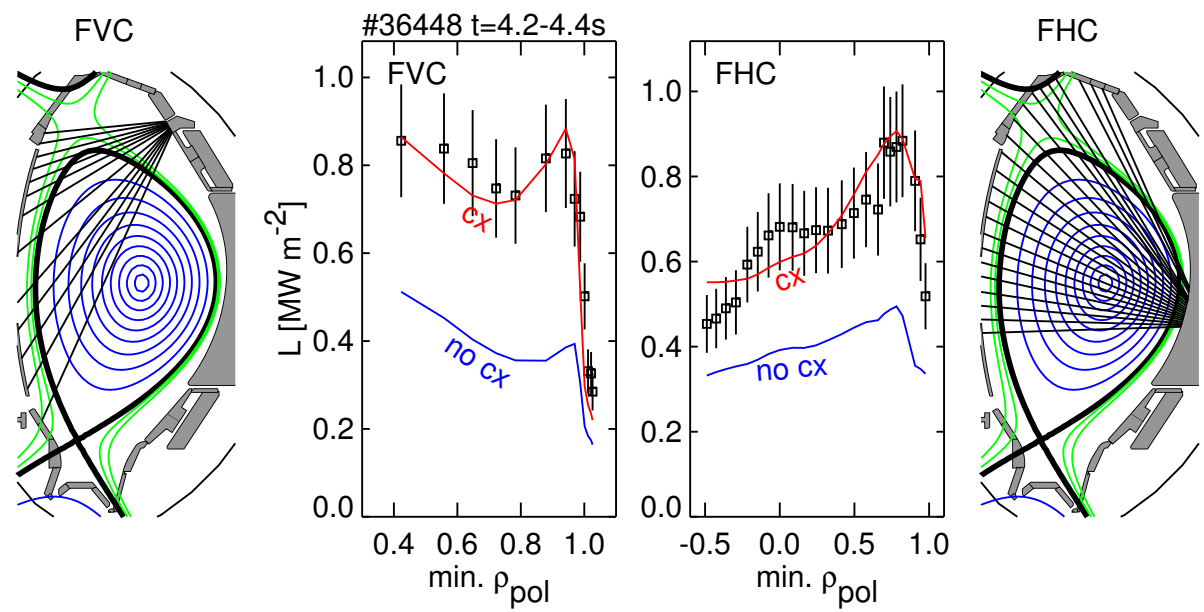

Figure 13. The radiances from two bolometer cameras observing the main plasma and the corresponding model values without (blue) and with (red) CX recombination for \#36448 $t=4.2-4.4 \mathrm{~s}$.

Fig.13 compares measured and modelled total radiances for the lines-of-sight of two bolometer cameras observing the main plasma. The red lines show the model with CX-reactions, where the height of the neutral density profile (the shape was fixed) was 
adapted to fit the measurement. Without CX-reactions the model delivers about a factor of 2 lower radiances for the same argon density profile.
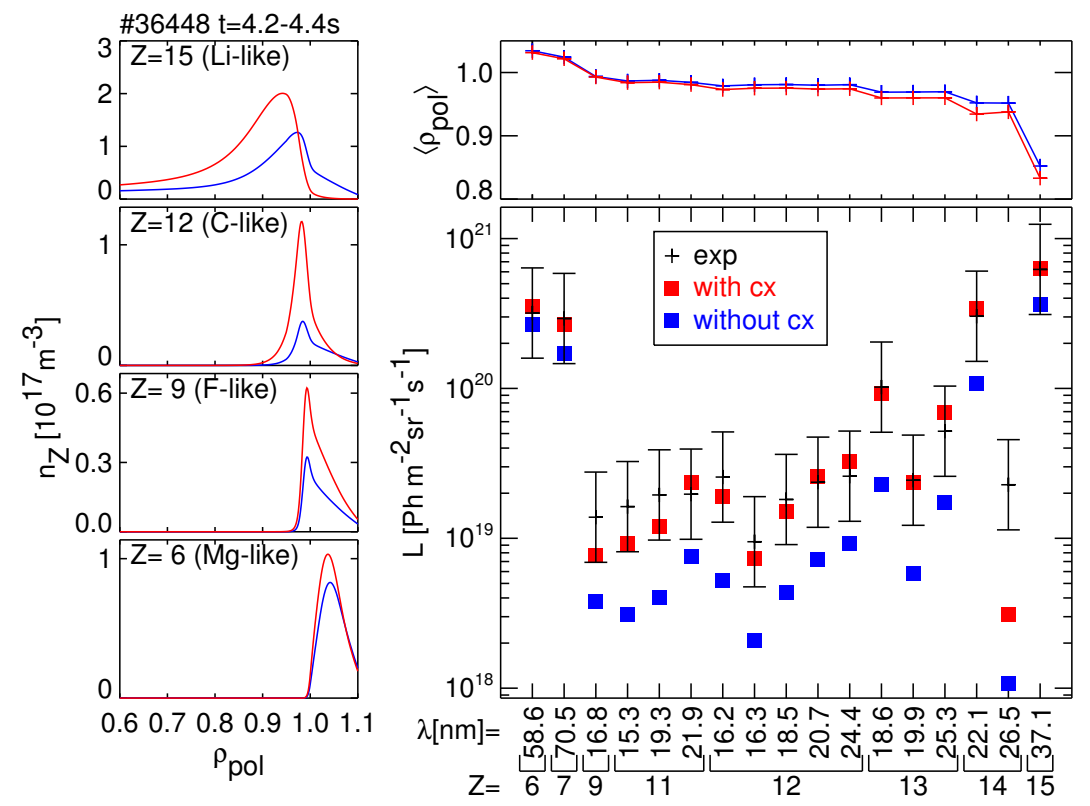

Figure 14. The radiances of 17 multiplets emitted by argon ions with charge $Z=6-15$ in the VUV range along a radial line-of-sight at the plasma equator and the corresponding model values without (blue) and with (red) CX recombination for \#36448 $t=4.2-4.4 \mathrm{~s}$. The upper graph shows the mean position of the emission and the boxes on the left display the density profiles for a number of argon ions which emit these VUV lines. The main difference between the models with and without CX is for lines emitted in the confined plasma close to the separatrix.

The SPRED spectrometer, which has a radial line-of-sight at the plasma equator, measures in the VUV region from 12 to $90 \mathrm{~nm}$. The spectrometer has been relatively calibrated by comparing VUV-lines and visible lines measured with a calibrated visible spectrometer on a similar line-of-sight for cases where both spectrometers could detect line emission from the same ion using $\mathrm{He}^{+}, \mathrm{B}^{2+}, \mathrm{C}^{2+}, \mathrm{C}^{3+}$, and $\mathrm{N}^{3+}$. In \#36448, many VUV-multiplets emitted by argon from charge stages between $Z=6$ (Mg-like) and $Z=15$ (Li-like) could be identified. The radiances of the multiplets which could be well fitted are shown in Fig.14. They are sorted by the charge of the emitting ion and for each ion by the central wavelength of the multiplet. For completeness, the spectroscopic notations of the lines are given in Tab.1.

Fortunately, there exist new atomic data sets from Bluteau [25] to perform generalised collisional-radiative modelling within the ADAS framework of codes [9]. Here, we used the type of photon emissivity coefficients, which assume statistical equilibrium between the fine-structure levels (in ADAS called LS-data) and an equilibration of the populations of the different spin systems (in ADAS called unresolved data). The total emitted photon rate of the multiplets has a contribution due to excitation and a small contribution due to recombination for the considered plasma 
Influence of $C X$-reactions on the radiation in the pedestal region at ASDEX Upgrade21

\begin{tabular}{|l|l|l|}
\hline ion & multiplet & $\lambda[\mathrm{nm}]$ \\
\hline ArVII & $2 \mathrm{p}^{6} 3 \mathrm{~s} 3 \mathrm{p}{ }^{1} \mathrm{P} \rightarrow 2 \mathrm{p}^{6} 3 \mathrm{~s}^{2}{ }^{1} \mathrm{~S}$ & 58.5 \\
ArVIII & $2 \mathrm{p}^{6} 3 \mathrm{p}^{2} \mathrm{P} \rightarrow 2 \mathrm{p}^{6} 3 \mathrm{~s}^{2} \mathrm{~S}$ & 70.5 \\
ArX & $2 \mathrm{~s} 2 \mathrm{p}^{6}{ }^{2} \mathrm{~S} \rightarrow 2 \mathrm{~s}^{2} 2 \mathrm{p}^{5}{ }^{2} \mathrm{P}$ & 16.8 \\
ArXII & $2 \mathrm{~s} 2 \mathrm{p}^{4}\left({ }^{3} \mathrm{P}\right){ }^{2} \mathrm{P} \rightarrow 2 \mathrm{~s}^{2} 2 \mathrm{p}^{3}{ }^{2} \mathrm{D}$ & 15.3 \\
ArXII & $2 \mathrm{~s} 2 \mathrm{p}^{4}\left({ }^{1} \mathrm{D}\right){ }^{2} \mathrm{D} \rightarrow 2 \mathrm{~s}^{2} 2 \mathrm{p}^{3}{ }^{2} \mathrm{D}$ & 19.3 \\
ArXII & $2 \mathrm{~s} 2 \mathrm{p}^{4}\left({ }^{3} \mathrm{P}\right){ }^{4} \mathrm{P} \rightarrow 2 \mathrm{~s}^{2} 2 \mathrm{p}^{3}{ }^{4} \mathrm{~S}$ & 21.9 \\
ArXIII & $2 \mathrm{~s} 2 \mathrm{p}^{3}{ }^{3} \mathrm{~S} \rightarrow 2 \mathrm{~s}^{2} 2 \mathrm{p}^{2}{ }^{3} \mathrm{P}$ & 16.2 \\
ArXIII & $2 \mathrm{~s} 2 \mathrm{p}^{3}{ }^{1} \mathrm{P} \rightarrow 2 \mathrm{~s}^{2} 2 \mathrm{p}^{2}{ }^{1} \mathrm{D}$ & 16.3 \\
ArXIII & $2 \mathrm{~s} 2 \mathrm{p}^{3}{ }^{1} \mathrm{D} \rightarrow 2 \mathrm{~s}^{2} 2 \mathrm{p}^{2}{ }^{1} \mathrm{D}$ & 18.5 \\
ArXIII & $2 \mathrm{~s} 2 \mathrm{p}^{3}{ }^{3} \mathrm{P} \rightarrow 2 \mathrm{~s}^{2} 2 \mathrm{p}^{2}{ }^{3} \mathrm{P}$ & 20.7 \\
ArXIII & $2 \mathrm{~s} 2 \mathrm{p}^{3}{ }^{3} \mathrm{D} \rightarrow 2 \mathrm{~s}^{2} 2 \mathrm{p}^{2}{ }^{3} \mathrm{P}$ & 24.4 \\
ArXIV & $2 \mathrm{~s} 2 \mathrm{p}^{2}{ }^{2} \mathrm{P} \rightarrow 2 \mathrm{~s}^{2} 2 \mathrm{p}{ }^{2} \mathrm{P}$ & 18.6 \\
ArXIV & $2 \mathrm{~s} 2 \mathrm{p}^{2}{ }^{2} \mathrm{~S} \rightarrow 2 \mathrm{~s}^{2} 2 \mathrm{p}{ }^{2} \mathrm{P}$ & 19.9 \\
ArXIV & $2 \mathrm{~s} 2 \mathrm{p}^{2}{ }^{2} \mathrm{D} \rightarrow 2 \mathrm{~s}^{2} 2 \mathrm{p}{ }^{2} \mathrm{P}$ & 25.3 \\
ArXV & $2 \mathrm{~s} 2 \mathrm{p}{ }^{1} \mathrm{P} \rightarrow 2 \mathrm{~s}^{2} \mathrm{~S}$ & 22.1 \\
ArXV & $2 \mathrm{p}^{2}{ }^{3} \mathrm{P} \rightarrow 2 \mathrm{~s} 2 \mathrm{p}{ }^{3} \mathrm{P}$ & 26.5 \\
ArXVI & $2 \mathrm{p}{ }^{2} \mathrm{P} \rightarrow 2 \mathrm{~s}{ }^{2} \mathrm{~S}$ & 37.1 \\
\hline
\end{tabular}

Table 1. Spectroscopic Notation of the used VUV-lines from argon and central wavelength of the multiplet.

region. For a multiplet emitted by an ion with charge $z$, the line radiance can be calculated with the help of the respective emissivity coefficient $p_{\text {exc }}$ and $p_{\text {rec }}$ by integration over the line-of-sight through the plasma.

$$
L=\int_{L O S} \epsilon d l=\frac{1}{4 \pi} \int_{L O S} n_{e}\left[n_{z} p_{e x c}\left(n_{e}, T_{e}\right)+n_{z+1} p_{r e c}\left(n_{e}, T_{e}\right)\right] d l
$$

Here, the recombination contributed less than a percent to the line emission. There are no atomic data, which would give the CX recombination into each of the excited levels of argon and allow for the calculation of photon emissivity coefficients from CX. Thus, $\mathrm{CX}$ is only considered via its effect on the ionisation balance, however, this is certainly by far the leading effect since the recombination contribution is so small.

The STRAHL model shows that the total radiation of the measured multiplets accounts for about half of the total line radiation emitted by these ion stages. Thus, an absolute intensity calibration of the VUV spectrometer can be obtained when comparing with the bolometric measuremnts. Since we know that the STRAHL calculation with CX explains the total radiation in the main plasma well, it also has to fit the individual lines measured by the SPRED that make up for a large fraction of the total radiation. Using this assumption, a single scaling factor was applied to the known relative sensitivity curve of the VUV spectrometer. The comparison between modelled and measured radiances is shown in Fig.14 using red squares for the case with CX and blue for the case without CX. Besides one outlier (ArXV at $\lambda=26.5 \mathrm{~nm}$ ), which is a 
factor of 7.4 too small, there is rather good agreement with the measurement when $\mathrm{CX}$ is on. Here, the mean deviation between model and measurement is $24 \%$ and the maximum deviation is $-44 \%$. When $\mathrm{CX}$ is off, the emission from $Z=5$ and 6 and from $Z=15$ are not much lower, but especially the lines from $Z=11$ and 12 , which come from the outer pedestal close to the separatrix, are much too low. The upper graph shows the average emission position of the lines for both cases, i.e. $\left\langle\rho_{\text {pol }}\right\rangle=\int \epsilon \rho_{\text {pol }} d l / \int \epsilon d l$. They are a bit shifted towards the core when CX is on, however, the higher radiances with CX are not due to this shift of the emitting shell into a region with higher $n_{e}$ and thus higher excitation and emission rates according to eq.(3) but due to a higher fractional abundance of the emitting ion. The densities of 4 stages with and without CX are displayed on the left side of Fig.14 and the largest difference is for $Z=12$, where the strongest deviation of the model without CX was found. For the Mg-like ion stage, which has its maximum in the SOL, the densities are almost equal. Even though the CX recombination is much larger than the electronic recombination for this stage, it does not influence the density distribution much. Rather it is governed by a balance of the ionisation rate and the radial transport, i.e. ions which are transported inward get ionised to higher stages, while most ions which move outwards do not recombine to lower ion stages during their travel time towards the plasma facing components. The ArXV line at $\lambda=26.5 \mathrm{~nm}$, which is not well represented by the model, is actually a ${ }^{3} \mathrm{P}-$ ${ }^{3} \mathrm{P}$ multiplet consisting of 6 lines between $25.48 \mathrm{~nm}$ and $27.59 \mathrm{~nm}$. The whole multiplet could be well fitted with prescribed line ratios assuming LS-coupling and we are sure that there is no wrong assignment of this multiplet.

\section{Projection to the pedestal radiation in ITER}

A simple projection of the CX effect to the much hotter pedestals in ITER was done. The test case was taken from a previous study on the tungsten transport in the ITER pedestal [26] with $T_{\text {sep }}=200 \mathrm{eV}, T_{p e d}=4.5 \mathrm{keV}, n_{e, s e p}=3 \times 10^{19} \mathrm{~m}^{-3}$ and $n_{e, p e d}=8 \times 10^{19} \mathrm{~m}^{-3}$ representing typical parameters for a DT-plasma at $I_{p}=15 \mathrm{MA}$ and $B_{T}=5.3 \mathrm{~T}$. We simply use $T_{e}=T_{i}$ and assume purely diffusive impurity transport to have a flat impurity density in the confined plasma. In Fig.15, the corresponding profiles are shown on the left side. Here, we have argon as impurity with a concentration of $0.1 \%$. The ionisation balance and the argon radiation is then calculated without and with CX recombination using the neutral deuterium density profile shown in the upper left box, which has $n_{D, \text { sep }}=2 \times 10^{16} \mathrm{~m}^{-3}$, i.e. a typical value from the investigated ASDEX Upgrade plasmas. Whether these values are a good assumption and sufficient to give enough fuelling in accordance with the density increase in the pedestal is outside the scope of this investigation.

On the right side of Fig.15, the upper box displays the profile of the mean argon charge (red with CX, blue without) which is shifted to lower ion stages with CX. This causes more radiation outside of $\rho_{\text {pol }}=0.9$ and the radiated power of argon inside the separatrix increases from $11 \mathrm{MW}$ to $21 \mathrm{MW}$ when switching on CX recombination. 

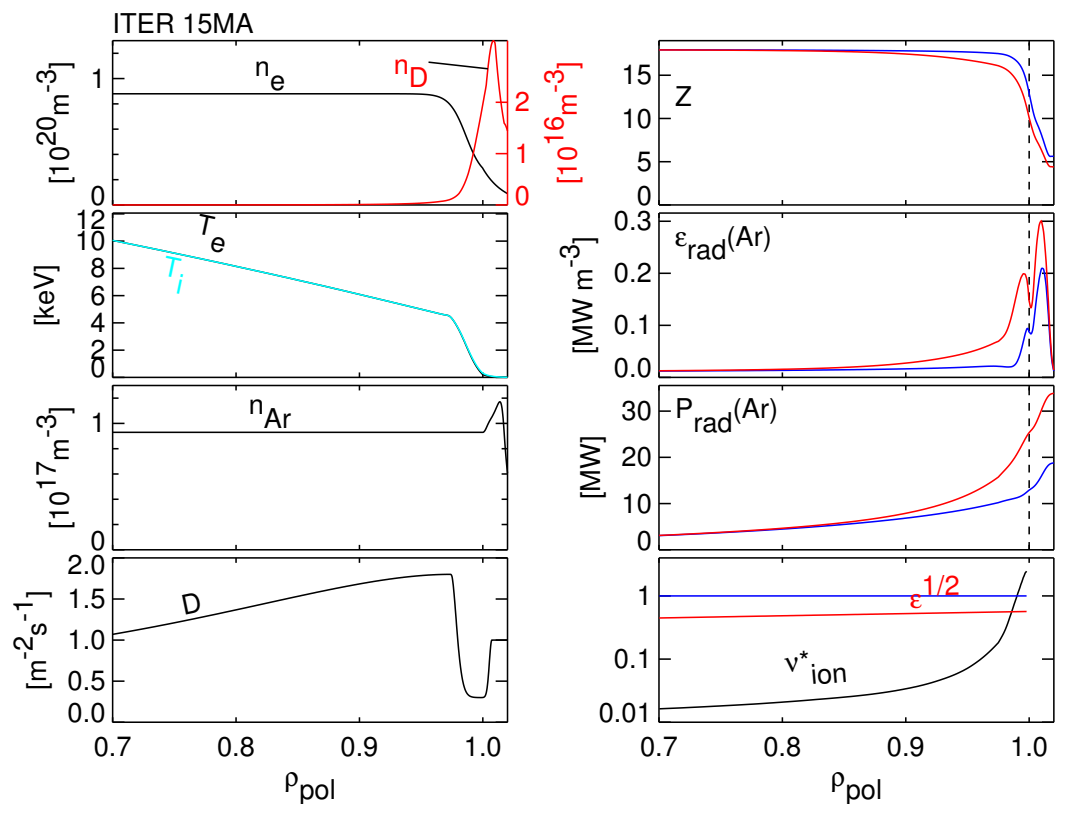

Figure 15. The radial profiles of $n_{e}, n_{D}, T_{e}=T_{i}$ and $n_{A r}$ for test calculations, whether CX recombination influences the Ar radiation in a hot ITER pedestal. With CX (red lines) the ionisation balance is shifted towards lower ion stages, which can better radiate and increase $P_{\text {rad,sep }}$ of Ar by about a factor of 2 .

The normalised ionisation rate from eq.(2) becomes larger than 1 for $\rho_{\text {pol }} \geq 0.99$ $\left(\Delta r_{\text {sep }} \leq 3.8 \mathrm{~cm}, T \leq 1.6 \mathrm{keV}\right)$ as can be seen in the lowest box on the right and the one dimensional treatment with a flux surface averaged neutral density is rather well justified for this estimate of the CX effect on pedestal radiation.

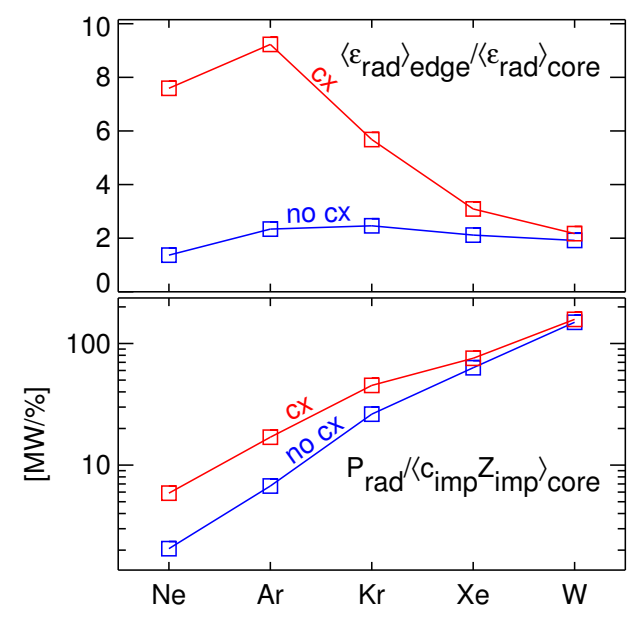

Figure 16. For the light elements up to $\mathrm{Kr}$, the ratio of the volume averaged edge and core radiation and the total radiated power inside the separatrix per fuel dilution in the core are benefiting from the CX-recombination in the pedestal region of ITER. For high-Z elements, the effect is small. 
This calculation has been performed for $\mathrm{Ne}$, Ar, Kr, Xe, and W, and volume averages of the impurity radiation in the core region and the edge region were computed. For the core, we used the radial range $0-1.2 \mathrm{~m}$ where $T$ is larger than $12 \mathrm{keV}$ and 2.1 $2.53 \mathrm{~m}$ for the edge where $T \leq 6 \mathrm{keV}$. The ratio of the averages, i.e. edge divided by core radiation, is shown in the upper graph of Fig.16 for each impurity with (red) and without (blue) CX. A high value is beneficial, since then a radiation loss can be obtained from edge radiation without invoking too much radiation loss in the core. Without $\mathrm{CX}$, the values are around 2 for all impurities and 1.4 for Ne. With CX, the values substantially increase for the low-Z elements up to Kr by factors $5.6(\mathrm{Ne}), 3.9$ (Ar), and 2.3 (Kr). The effect is small for Xe and almost zero for W. For light elements, mainly the dilution of the fuel ions is important since it reduces the fusion power. Thus, the lower box shows the ratio of the total radiated power within the separatrix dived by the volume average of $\sum_{z} Z n_{z} / n_{e}$ in the core region. The radiated power per dilution strongly increases with impurity charge and is highest for W. Again, the CX recombination in the pedestal does not change this value for high-Z elements, but allows for higher radiation for the elements up to Kr. The values increase for Ne, Ar, and Kr by a factor of 2.9, 2.5, and 1.7, respectively. However, the increase is not so strong that a higher-Z element like Xe could be replaced by Kr. The low influence on the radiation of the high-Z elements is caused by a lower increase of the recombination rates and the fact that neighbouring ion stages in the pedestal region can radiate about equally well.

\section{Conclusions}

The major finding of the study described in this paper is that charge exchange from neutral hydrogen onto impurity ions leads to strong modifications of the ionisation equilibrium of neon and argon in the pedestal region of H-mode plasmas in ASDEX Upgrade. CX causes a higher abundance of lower ionised stages and a concomitant increase of the pedestal radiation from neon and argon. In the investigated cases, the total radiated power inside the separatrix increases by a factor of 5 for neon and 2.2 for argon due to the shifted ionisation balance. A simple projection of these results to the much hotter pedestals in ITER revealed that the neutral density can also substantially increase the pedestal radiation in ITER but only for the lighter elements $\mathrm{Ne}$, Ar, and $\mathrm{Kr}$, while CX has a low influence on the radiation from Xe and W. A better understanding or even control of the neutral D distribution inside the separatrix appears mandatory for regimes where tailoring of the pedestal pressure profile is important.

\section{Acknowledgement}

This work has been carried out within the framework of the EUROfusion Consortium and has received funding from the Euratom research and training programme 2014-2018 and 2019-2020 under grant agreement No 633053. The views and opinions expressed herein do not necessarily reflect those of the European Commission. 
Influence of $C X$-reactions on the radiation in the pedestal region at ASDEX Upgrade25

\section{References}

[1] Suckewer S et al. 1980 Phys. Rev. A $\mathbf{2 2 ( 2 )}$ 725-731 URL https://link.aps.org/doi/10.1103/PhysRevA.22.725

[2] Hulse R A et al. 1980 Journal of Physics B: Atomic and Molecular Physics 13 3895-3907 URL https://doi.org/10.1088/0022-3700/13/19/026

[3] Puiatti M E et al. 1981 Plasma Physics 23 1075-1092 URL https://doi.org/10.1088/0032-1028/23/11/007

[4] Rice J E et al. 1986 Phys. Rev. Lett. 56(1) 50-53 URL https://link.aps.org/doi/10.1103/PhysRevLett.56.50

[5] Viezzer E et al. 2011 Plasma Physics and Controlled Fusion 53035002 URL http://stacks.iop.org/0741-3335/53/i=3/a=035002

[6] Isler R C 1994 Plasma Physics and Controlled Fusion $\mathbf{3 6}$ 171-208 URL https://doi.org/10.1088/0741-3335/36/2/001

[7] Viezzer E et al. 2012 Review of Scientific Instruments 83103501 (pages 11) URL http://link.aip.org/link/?RSI/83/103501/1

[8] McDermott R M et al. 2018 Plasma Physics and Controlled Fusion 60095007 URL http://stacks.iop.org/0741-3335/60/i=9/a=095007

[9] Summers H P 2004 Atomic data and analysis structure users manual 2.6, http://www.adas.ac.uk/manual.php

[10] Dux R et al. 2012 in Proc. of the 39th EPS Conference on Plasma Physics, Stockholm, Sweden, 2012 p P2.049 URL http://ocs.ciemat.es/EPSICPP2012PAP/pdf/P2.049.pdf

[11] DUX R 2006 STRAHL User Manual Tech. Rep. 10/30 IPP, Garching, Germany URL http://hdl . handle.net/11858/00-001M-0000-0027-0DB8-4

[12] Janev R K and Smith J J 1993 Atomic and Plasma Material Interaction Data for Fusion (Atomic and Plasma Material Interaction Data for Fusion no 4) (Vienna: INTERNATIONAL ATOMIC ENERGY AGENCY) URL https://www.iaea.org/publications/1839/atomic-and-plasma-material-interaction-data-for-fusion

[13] Eckstein W 2009 Tech. Rep. IPP 17/12 Max-Planck Institut für Plasmaphysik, Garching, Germany URL http://hdl.handle.net/11858/00-001M-0000-0026-F340-E

[14] Kallenbach A et al. 2020 Developments towards an ELM-free DEMO pedestal radiative cooling scenario using argon seeding in ASDEX Upgrade, submitted to Nuclear Fusion

[15] Pütterich $\mathrm{T}$ et al. 2011 Journal of Nuclear Materials 415 S334-S339 URL https://doi.org/10.1016/j.jnucmat.2010.09.052

[16] Dux R et al. 2011 Nuclear Fusion 51053002 URL http://stacks . iop.org/0029-5515/51/i=5/a=053002

[17] Gil L et al. 2020 Nuclear Fusion 60054003 URL https://doi.org/10.1088/1741-4326/ab7d1b

[18] Odstrčil $\mathrm{T}$ et al. 2016 Review of Scientific Instruments $\mathbf{8 7} 123505$ URL http://dx.doi.org/10.1063/1.4971367

[19] Henke B et al. 1993 Atomic Data and Nuclear Data Tables 54181 - 342 ISSN 0092-640X URL http://www.sciencedirect.com/science/article/pii/S0092640X83710132

[20] Anton $\mathrm{M}$ et al. 1995 Review of Scientific Instruments $\mathbf{6 6} \quad 3762-3769$ URL https://doi.org/10.1063/1.1145434

[21] Karzas W J and Latter R 1961 The Astrophysical Journal Supplement Series 6 167-212

[22] Post D et al. 1977 Atomic Data and Nuclear Data Tables 20397 - 439 ISSN 0092-640X URL http://www.sciencedirect.com/science/article/pii/0092640X77900262

[23] Pütterich $\mathrm{T}$ et al. 2008 Plasma Physics and Controlled Fusion 50085016 URL http://stacks.iop.org/0741-3335/50/085016

[24] McDermott R M et al. 2020 Development of $\mathrm{Ar}^{+16}$ charge exchange recombination spectroscopy measurements at ASDEX Upgrade, submitted to Nuclear Fusion

[25] Bluteau M M 2019 Fundamental atomic data and prototype techniques for a generalised collisionalradiative model of medium-weight elements in fusion and astrophysical plasmas, $\mathrm{PhD}$ thesis Uni- 
Influence of $C X$-reactions on the radiation in the pedestal region at ASDEX Upgrade26 versity of Strathclyde Glasgow URL http://www.adas.ac.uk/theses/bluteau_thesis.pdf

[26] Dux R et al. 2014 Plasma Physics and Controlled Fusion $\mathbf{5 6} 124003$ URL https://doi.org/10.1088/0741-3335/56/12/124003 